\title{
Fingerprinting of Natural Product by Eastern Blotting Using Monoclonal Antibodies
}

\author{
Hiroyuki Tanaka, ${ }^{1}$ Waraporn Putalun, ${ }^{2}$ and Yukihiro Shoyama ${ }^{3}$ \\ ${ }^{1}$ Faculty of Pharmaceutical Science, Kyushu University, 3-1-1 Maidashi, Higashiku, Fukuoka 812-0855, Japan \\ ${ }^{2}$ Faculty of Pharmaceutical Science, Khon Kaen University, Khon Kaen 40002, Thailand \\ ${ }^{3}$ Faculty of Pharmaceutical Science, Nagasaki International University/2825-7 Huis Ten Bosch, Sasebo, Nagasaki 859-3298, Japan \\ Correspondence should be addressed to Yukihiro Shoyama, shoyama@niu.ac.jp
}

Received 18 May 2011; Accepted 9 November 2011

Academic Editor: Irena Vovk

Copyright (๑) 2012 Hiroyuki Tanaka et al. This is an open access article distributed under the Creative Commons Attribution License, which permits unrestricted use, distribution, and reproduction in any medium, provided the original work is properly cited.

\begin{abstract}
We succeeded in developing the fingerprint of natural product by eastern blotting using monoclonal antibodies. After developing and separating them on a TLC plate, solasodine glycosides are oxidized by $\mathrm{NaIO}_{4}$ and reacted with a protein to give conjugates which are recognized with anti-solamargine monoclonal antibody (MAb). Anti-solamargine MAb having wide cross-reactivity can stain and detect all solasodine glycosides by fingerprint. Different sensitivity between solamargine and solasonine was observed. The detection limit was $1.6 \mathrm{ng}$ of solasonine. The hydrolysed products of solamargine were determined by fingerprint of eastern blotting compared to their Rf values depending on the sugar number. Fingerprint by eastern blotting using anti-ginsenoside Rb1 $\mathrm{MAb}$ distinguished the formula containing ginseng prescribed in traditional Chinese medicine. By double-staining of ginsenosides it is possible to suggest that the staining color shows the pharmacological activity, such as the purple bands indicate ginsenosides having stimulation activity, and the blue color indicated compound like ginsenosides possessed the depression affect for the central nervous system (CNS), respectively.
\end{abstract}

\section{Introduction}

In the recent rapid development of the molecular biosciences and their biotechnological applications, immunoassay systems using monoclonal antibody (MAb) against drugs and small molecular weight bioactive compounds have become an important tool for studies on receptor binding analysis, enzyme assay, and quantitative and/or qualitative analytical techniques in animals or plants; owing to their specific affinity. Previously we prepared various kinds of MAb against natural products like forskolin [1], solamargine [2], crocin [3], marihuana compound [4], opium alkaloids [5], ginsenosides [6, 7], berberine [8], sennosides [9], paeoniflorin [10], glycyrrhizin [11, 12], ginkgolic acid [13], aconitine alkaloid [14], baicalin [15], and so on, and developed individual competitive enzyme-linked immunosorbent assay (ELISA) as a high sensitive, specific, and simple methodology.

Western blotting is widely used as an immunostaining technique to detect high-molecular compounds like peptides and proteins based upon an antigen-antibody reaction. However, low-weight molecular compounds had not been previously analyzed by western blotting. We succeeded the blotted staining of solasodine glycoside on PVDF membrane using $\mathrm{MAb}$ after developed and separated solasodine glycosides on TLC and called as new-western blotting [16]. From this evidence, we applied this new methodology to licorice glycoside, glycyrrhizin, and named it as eastern blotting [12] following ginsenosides [17-19] and saikosaponin [20], and so on. In this paper, we introduce the fingerprint of a steroidal alkaloid glycoside, solasodine glycoside and ginseng saponin, ginsenosides by eastern blotting using MAbs.

\section{Materials and Methods}

2.1. Preparation of Anti-Solamargine MAb. A hybridoma producing $\mathrm{MAb}$ reactive to solamargine was obtained by the general procedure in our laboratory and classified into IgG2b which had $\mathrm{k}$ light chains [2]. The reactivity of 
IgG type MAb, SMG-BD9, was tested by varying antibody concentration and by performing a dilution curve. The antibody concentration was selected for competitive ELISA. The MAb following competition was bound to polystyrene microtiter plates precoated with solamargine HSA. Under these conditions, the full measuring range of the assay extended from 20 to $400 \mathrm{ng} / \mathrm{mL}$ [2].

Anti-ginsenoside Rb1 [6] and Rg1 [7] were also prepared by the same ways in our laboratory.

2.2. Fingerprint by Eastern Blotting [16]. Solasodine glycosides were developed on a TLC plate. The TLC plate was covered with the PVDF membrane and blotted by short heating. The blotted PVDF membrane was dipped in water containing $\mathrm{NaIO}_{4}$ under stirring at room temperature for $1 \mathrm{hr}$. After washing with water, a carbonate buffer containing BSA was added and stirred at room temperature for $3 \mathrm{hr}$. The PVDF membrane was washed twice with phosphate buffer for $5 \mathrm{~min}$, and then washed with water. The PVDF membrane was immersed in anti-solamargine MAb, stirred at room temperature for $1 \mathrm{hr}$. After washing the PVDF membrane twice with phosphate buffer and water, a 1000-fold dilution of peroxidase-labeled goat anti-mouse IgG in phosphate buffer ( $\mathrm{pH} 7.2)$ was added and stirred at room temperature for $1 \mathrm{hr}$. The PVDF membrane was washed twice with phosphate buffer and water, then exposed to 4-choloro-1naphthol $(1 \mathrm{mg} / \mathrm{mL})-\mathrm{H}_{2} \mathrm{O}_{2}(0.03 \%)$ in phosphate buffer (pH 7.2), and eastern blotting was stopped by washing with water. The immunostained PVDF membrane was allowed to dry.

2.3. Hydrolysis of Solasodine Glycosides and Stained by $\mathrm{H}_{2} \mathrm{SO}_{4}$, Dragendorff, and Eastern Blotting. Solamargine and solasonine were hydrolyzed by using $1 \mathrm{M} \mathrm{HCl}$ and heated for 10, 20, 30,60 , and 90 minutes, respectively. The hydrolyzed products were applied on three silica gel TLC plates. Developments were made by using $\mathrm{CHCl}_{3} / \mathrm{MeOH} / \mathrm{NH}_{4} \mathrm{OH}(7: 2.5: 1)$ as mobile phase. Two plates were sprayed with $\mathrm{H}_{2} \mathrm{SO}_{4}$ and Dragendorff reagent, respectively. But the third TLC plate was transferred to a PVDF membrane by heating and stained as indicated above.

\section{Results and Discussion}

3.1. Fingerprint of Solasodine Glycosides by Eastern Blotting. The natural resources of adrenocortical hormones and sex hormones, which are mainly obtained from diosgenin, are becoming rare. The most important feature of solasodine is that it can be converted to dehydropregnenolone. Solasodine is found with a series of sugar residues attached to the oxygen at the $\mathrm{C}-3$ position. By far the most common forms are the triglycosides, solamargine being predominant. Therefore, the steroidal alkaloid glycosides of the solasodine type like solamargine have become important as a starting material for the production of steroidal hormones in the pharmaceutical and medicinal areas. Rapid, simple, highly sensitive, and reproducible assay systems are required for a large number of plants, and a limited small amount of samples. In order to select the strain of higher yielding steroidal alkaloid

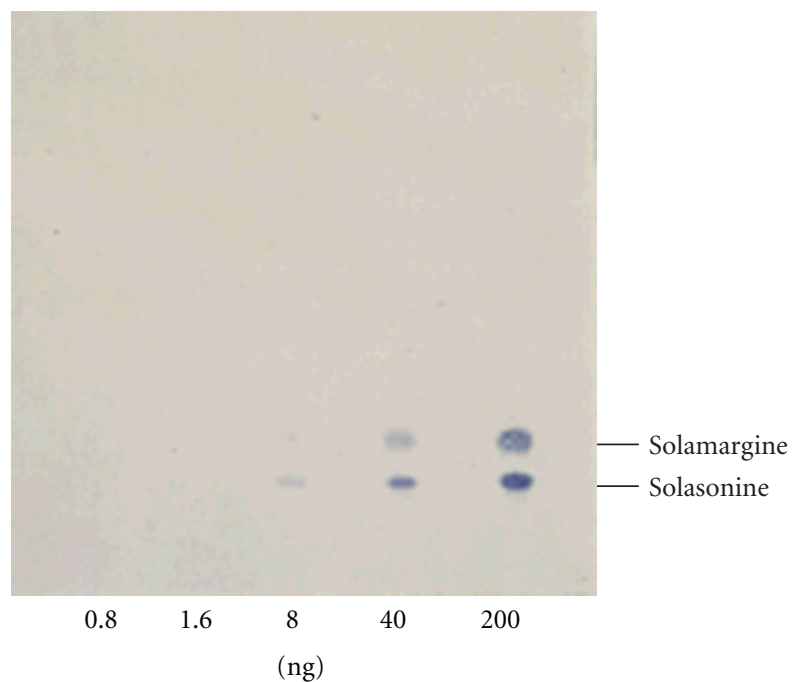

FIGURE 1: Fingerprint of solamargine and solasonine by eastern blotting.

glycosides in the resources of Solanum species we prepared an anti-solamargine $\mathrm{MAb}$ which was unique having a wide cross-reactivity [2].

Clear blue spots appeared on PVDF membrane as shown in Figure 1, the immunostains of solamargine and solasonine were observed. Lane 1-5 demonstrated the concentrations of both alkaloids, $0.8,1.6,8,40$, and $200 \mathrm{ng}$, respectively. Different sensitivities between solamargine and solasonine were observed in individual concentrations, and the sensitivity of solasonine was somewhat higher than that of solamargine. The detection limit was $1.6 \mathrm{ng}$ of solasonine. We succeeded to separate the function of small molecule compounds such as solasodine glycosides into a part of epitope and fixing on the membrane [16]. When glycosides are treated by $\mathrm{NaIO}_{4}$, the sugar part is cleaved to release an aldehyde group which is prepared a Schiff base with protein and then fixed to PVDF membrane. On the other hand, an epitope can be detected by $\mathrm{MAb}$ as described in Figure 2.

In order to confirm the preparation of solamargine-BSA conjugate on the PVDF membrane, the band corresponding to the solamargine-BSA conjugate was assessed by MALDI mass spectrometry. A broad peak $[\mathrm{M}+\mathrm{H}]+$ of solamargineBSA conjugate appeared at around m/z 69043 in MALDI mass spectrometry demonstrating that at least 1-2 molecules of solamargine had combined [6] (data not shown). Therefore, it became clear that the sugar moiety which was conjugated with BSA is necessary in this staining system. Moreover, we confirmed the above evidence that solasodine glycoside-protein conjugate is necessary for the eastern blotting because solasodine which has no sugar moiety in a molecule cannot be stained by eastern blotting although solasodine can be stained by $\mathrm{H}_{2} \mathrm{SO}_{4}$ and Dragendorff reagent (see Figures 3(b) and 3(c)).

As an application of finger printing by newly established eastern blotting, the hydrolysis pathway of solamargine was surveyed by eastern blotting using anti-solamargine MAb. Solamargine was hydrolyzed using $1 \mathrm{M} \mathrm{HCl}$ for 10,20 , 


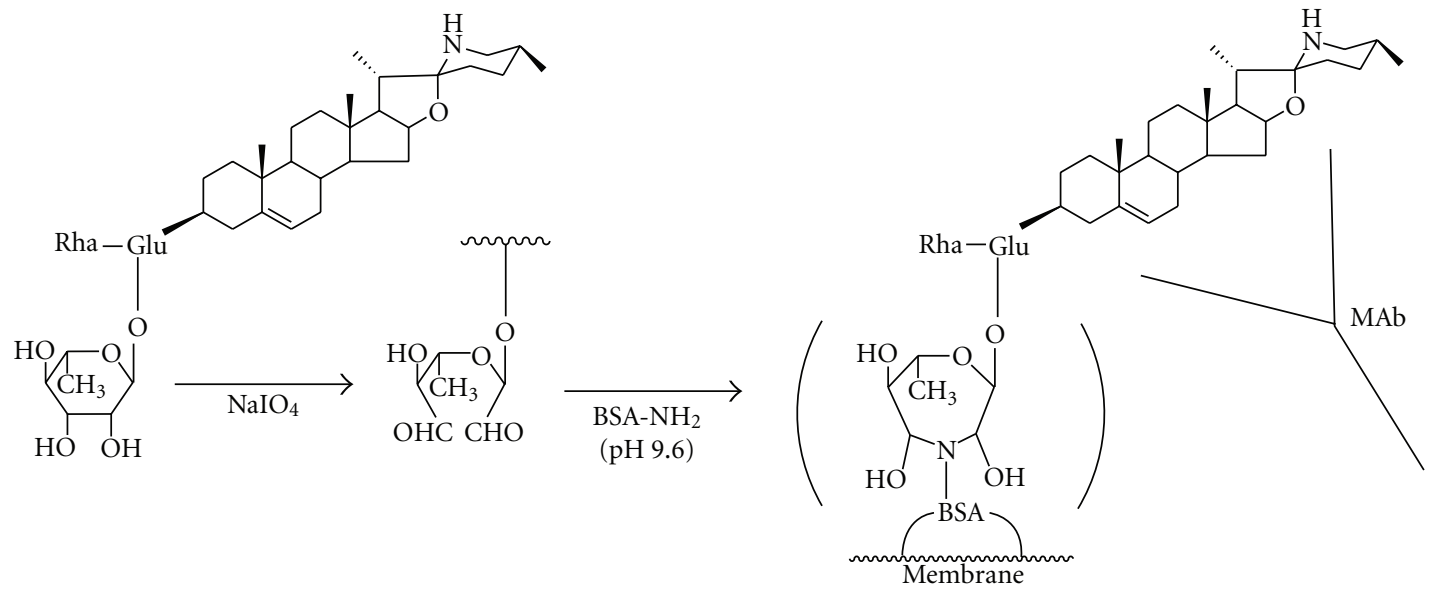

FIGURE 2: Staining mechanism of eastern blotting.
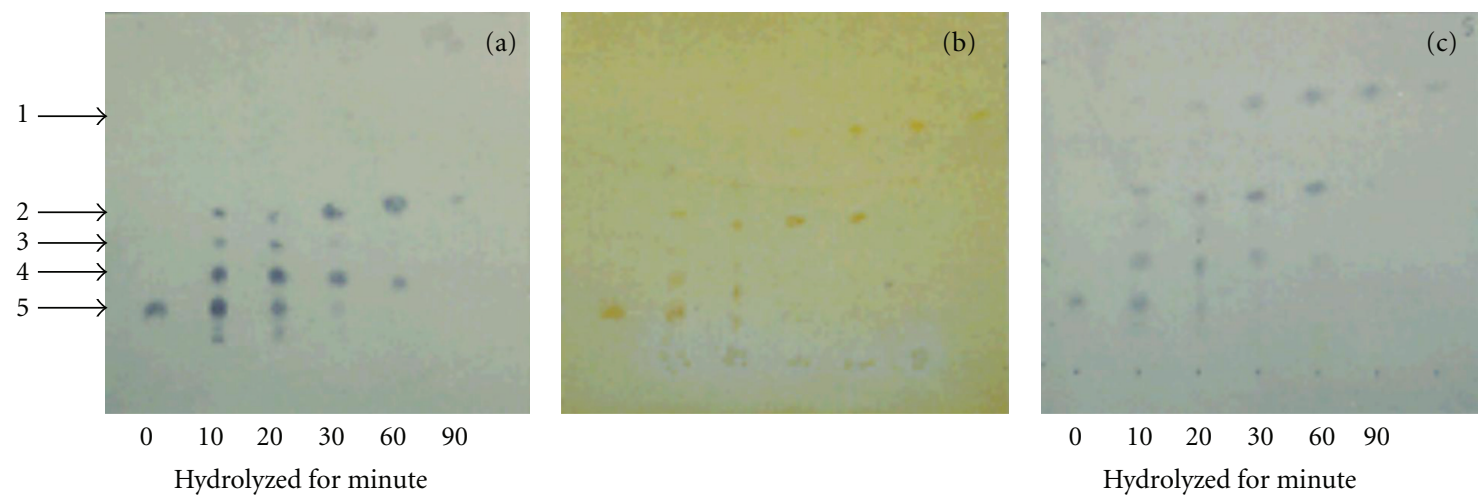

(1) Solasodine

(2) 3-O- $\beta$-D-glucopyranosyl solasodine

(3) L-rhamnosyl-(1-4)-O-3- $\beta$-D-glucopyranosyl solasodine (khasianine)

(4) L-rhamnosyl-(1-2)-O-3- $\beta$-D-glucopyranosyl solasodine

(5) Solamargine

Figure 3: Fingerprint of solasodine glycosides by eastern blotting (a), Dragendorff reagent (b), and sulfuric acid (c).

30, 60, and 90 minutes, respectively. The hydrolyzed products were developed by three silica gel TLC plates using $\mathrm{CHCl}_{3} / \mathrm{MeOH} / \mathrm{NH}_{4} \mathrm{OH}$ solvent system. Two plates were sprayed and colored with $\mathrm{H}_{2} \mathrm{SO}_{4}$ and Dragendorff reagent, individually. One TLC plate was blotted to a PVDF membrane and stained by eastern blotting. Clear blue spots appeared as shown in Figure 3. Figure 3(a) shows the eastern blotting of solasodine glycosides. Lanes 1-6 demonstrate solamargine, 10, 20, 30, 60, and 90 minutes hydrolyzed products, individually, solasodine, 3-O- $\beta$-D-glucopyranosyl solasodine, L-rhamnosyl-(1-4)-O-3- $\beta$-D-glucopyranosyl solasodine, L-rhamnosyl-(1-2)-O-3- $\beta$-D-glucopyranosyl solasodine, and solamargine, respectively. This fingerprinting phenomenon agreed with the structural confirmation of solasodine glycosides elucidated by ${ }_{13} \mathrm{C}$ NMR [21]. When compared with the sensitivity of fingerprinting regarding solasodine glycosides, the eastern blotting was more sensitive than those stained by $\mathrm{H}_{2} \mathrm{SO}_{4}$ (Figure 3(b)) and Dragendorff (Figure 3(c)).
We also analyzed the fingerprinting of Solanum species as shown in Figure 4 . The profile stained by $\mathrm{H}_{2} \mathrm{SO}_{4}$ (Figure 4(b)) indicated the complicated pattern which is not limited to solasodine glycosides compared to the eastern blotting profile (Figure 4(a)). Major solasodine glycoside are solasonine, solamargine, and khasianine as indicated in Figure 4. From the survey of solasodine glycoside we decided that $S$. khasianum is an important resource of adrenocortical hormones and sex hormones.

Moreover, we succeeded to breed the strain of higher yielding solasodine glycoside by inducing the single chain Fv gene cloned from anti-solamargine MAb into Solanum khasianum plant [22].

3.2. Fingerprint of Ginseng Saponin, Ginsenoside, by Eastern Blotting. Ginseng, the crude drug of Panax ginseng is one of the most important natural medicine in many countries. It has been used to enhance stamina and capacity to cope with fatigue and physical stress, and as a tonic against 


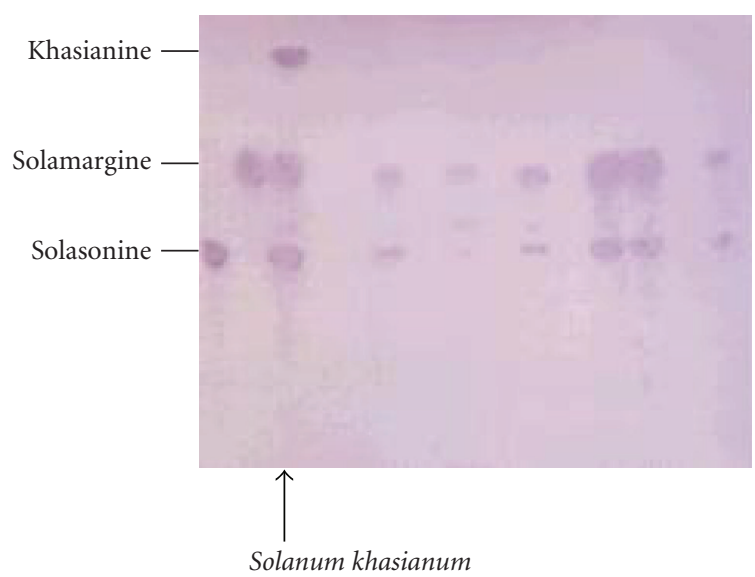

(a)

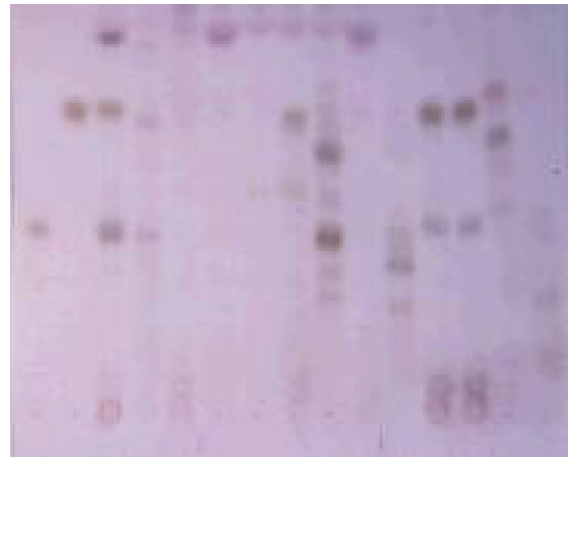

(b)

FIgURE 4: The eastern blotting (a) and TLC stained with sulfuric acid (b) of solasodine glycosides from Solanum spp.

cancers, disturbances of the central nervous system (CNS), hypothermia, carbohydrate and lipid metabolism, immune function, the cardiovascular system, and radioprotection [23]. They contain more than 50 kinds of dammarane and oleanane saponins considered to be pharmacologically active components. Ginsenoside Rb1 is a main saponin in ginseng. However, since the concentration varies in the ginseng root or the root extract depending on the method of extraction, subsequent treatment, or even the season of its collection, standardization of quality is required. For this purpose we have prepared anti-ginsenoside Rb1 [6] and Rg1 MAbs [7].

Cross-reactivity is the most important factor in determining the value of an antibody. Since the ELISA for ginsenoside $\mathrm{Rb} 1$ was established for phytochemical investigations involving crude plant extract, the assay specificity was checked by determining the cross-reactivity of the MAb with various related compounds. The cross-reactivity data of MAb that was obtained were examined by competitive ELISA and calculated using picomole of ginsenoside Rb1. The cross-reactivity of ginsenoside Rc and Rd which possess a diglucose moiety attached to the C-3 hydroxy group were weak compared to ginsenoside Rb1, 0.024 and $0.020 \%$, respectively. Ginsenoside Re and Rg1 showed no crossreactivity (less than $0.005 \%$ ). It is evident that the MAb reacted only with a small number of structurally related ginsenoside $\mathrm{Rb} 1$ molecules very weakly and did not react with other steroidal compounds like glycyrrhizin, digitoxin, tigogenin, tigonin, and solamargine. Anti-ginsenoside Rg1 MAb [7] and Re [18] have been prepared and set up their ELISA. Anti-ginsenoside Rg1 MAb was also high specific like anti-ginsenoside Rb1. On the other hand, anti-ginsenoside Re MAb showed wide cross-reactivity. Therefore, this MAb can be used for the analysis for the total ginsenoside concentration.

Figure 5 shows the $\mathrm{H}_{2} \mathrm{SO}_{4}$ staining and eastern blotting of ginsenoside standards and the traditional Chinese medicine (TCM) using anti-ginsenoside Rb1 MAb. It is impossible to determine the ginsenosides by TLC-staining by $\mathrm{H}_{2} \mathrm{SO}_{4}$ as indicated in Figure $5(\mathrm{a})$. On the other hand, clear staining of ginsenoside $\mathrm{Rb} 1$ occurred by eastern blotting (Figure 5(b)). Furthermore, it became evident that kikyoto and daiokanzoto prescriptions related to TCM prescription that did not contain ginseng indicating no band of ginsenoside $\mathrm{Rb} 1$. The eastern blotting method was considerably more sensitive than that of $\mathrm{H}_{2} \mathrm{SO}_{4}$ staining. The $\mathrm{H}_{2} \mathrm{SO}_{4}$ staining detected all standard compounds. The Eastern blotting indicated only limited staining of ginsenoside Rb1, $\mathrm{Rc}$, and Rd, of which cross-reactivities were under $0.02 \%$. We suggest that an aglycon, protopanaxadiol, and a part of the sugars may be of importance to the immunization and may function as an epitopes for the structure of ginsenosides. In addition, it is suggested that the specific reactivity of sugar moiety in the ginsenoside molecule against anti-ginsenoside $\mathrm{Rb} 1 \mathrm{MAb}$ may be modified by the $\mathrm{NaIO}_{4}$ treatment of ginsenosides on the PVDF membrane causing ginsenoside Rc and Rd to become detectable by eastern blotting.

When the mixture of anti-ginsenoside $\mathrm{Rb} 1$ and $\mathrm{Rg} 1$ MAbs and the pair of substrates were tested for staining of ginsenosides, all ginsenosides, ginsenoside-Rb1,-Rc,-Rd,-Re, and -Rg1 were stained blue although the purple color staining for ginsenoside $\mathrm{Rg} 1$ was expected because 3amino-9-ethylcarbazole and 4-chloro-1-naphotol might be different. Therefore, we performed successive staining of the membrane using anti-ginsenoside $\operatorname{Rg} 1$ and then antiginsenoside Rb1. Finally, we succeeded: the double-staining of ginsenosides indicating that ginsenoside Rg1 and ginsenoside Re were stained purple and the other blue, as indicated in Figure 6. From this result, both antibodies can distinguish individual aglycon protopanaxatriol and protopanaxadiol. For this application, the crude extract of various Panax species were analyzed by the newly developed double-staining system. Major ginsenosides can be determined clearly by the double staining method indicated in Figure 6 as the fingerprint of ginsenosides. Therefore, it is possible to suggest that the staining color shows the pharmacological activity, such as the purple bands indicate ginsenosides having stimulation activity for the CNS. On the other hand, the blue color indicates ginsenosides possessing the depression affect for 


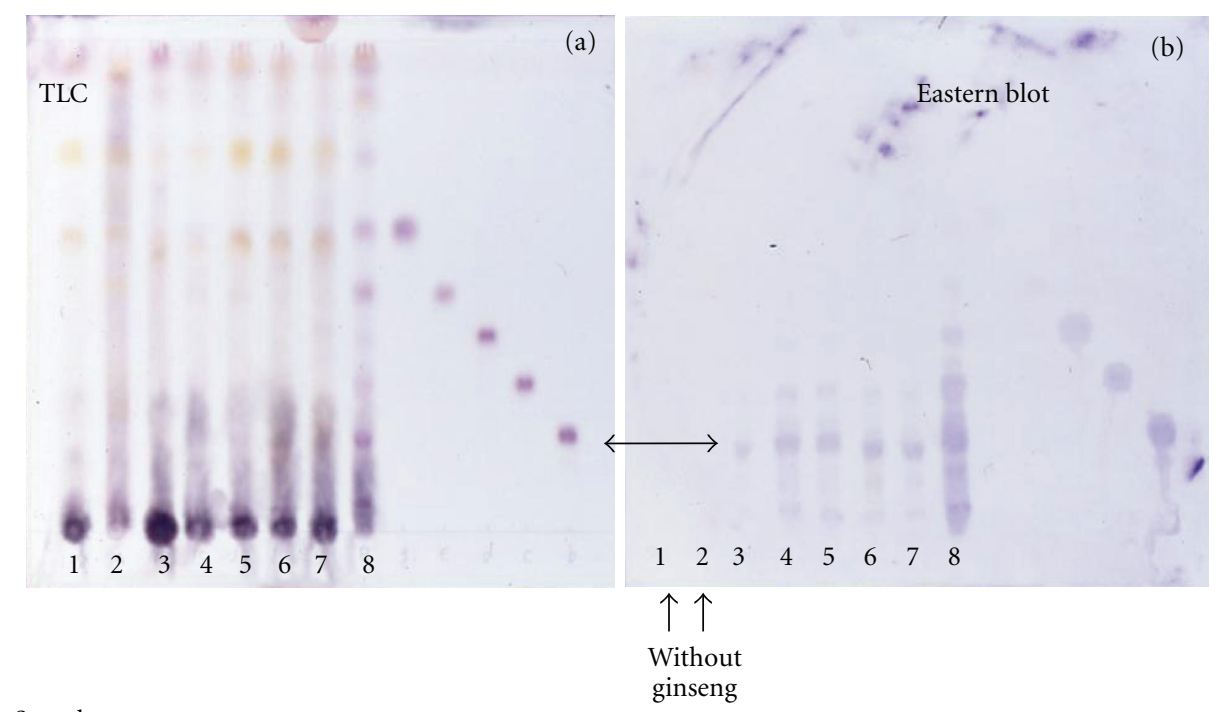

Samples
(1) Kikyoto
(2) Daiokanzoto
(3) Ninjinyoeito
(4) Shikunshito
(5) Ninjinto
(6) Hangeshashinto
(7) Shosaikoto
(8) Crude extract of ginseng

Samples 1 and 2 do not contain ginseng.

Standard of ginsenosides indicated ginsenoside-Rg1, -Re, -Rd, -Rc, and -Rb1 from upper.

FIGURE 5: TLC stained with sulfuric acid (a) and eastern blotting (b) of ginsenosides in traditional Chinese medicine (TCM) prescriptions by anti-ginsenoside Rb1 MAb.

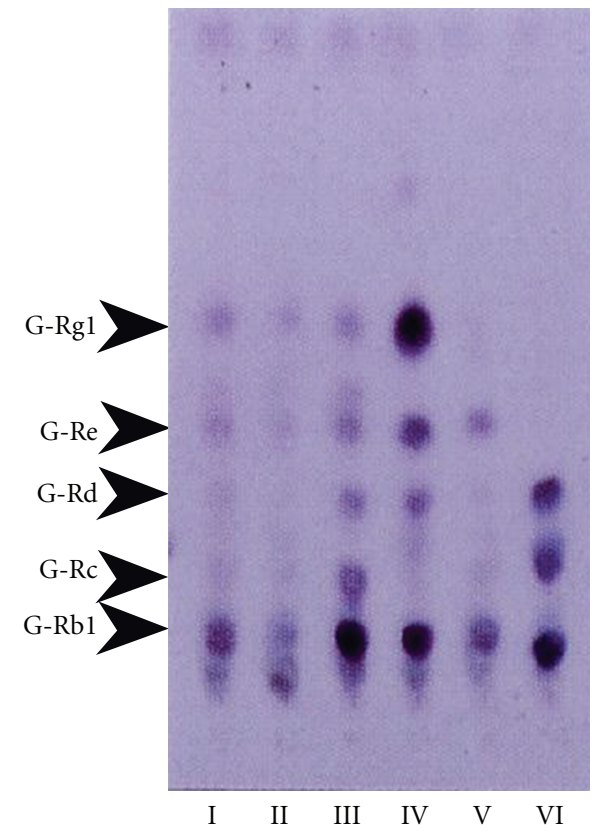

(a)

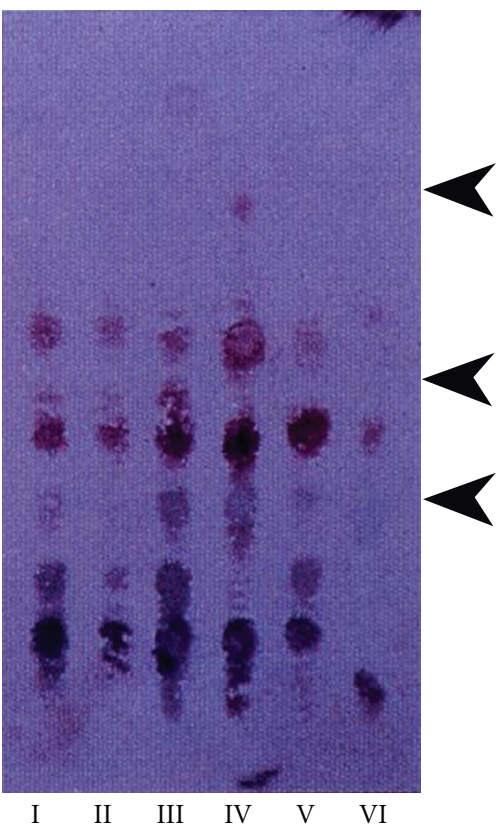

(b)

FIGURE 6: Double-staining of eastern blotting for ginsenosides contained in various ginseng using anti-ginseenoside-Rb1 and antiginsenoside-Rg1 monoclonal antibodies. (a) TLC profile stained by sulfuric acid, (b) Eastern blotting by anti-ginsenoside-Rb1 and antiginseside-Rg1 monoclonal antibodies. I, II, III, IV, V, and VI indicated white ginseng, red ginseng, fibrous ginseng (Panax ginseng), Panax notoginseng, Panax quinquefolius, and Panax japonicus, respectively. Upper purple color spots and lower blue color spots were stained by anti-ginsenoside-Rg1 and anti-ginsenoside-Rb1 monoclonal antibodies, respectively. 
the CNS. Moreover, the Rf value of ginsenosides roughly suggests the number of sugars attached to the aglycon. Both analyses make it possible to identify which aglycon attaches and how many sugars combine, leading to the structure of ginsenosides. In fact, three kinds of ginsenosides possessing protopanaxadiol-ginsenoside Rh1, Rf, and 20-Ogluco-ginsenoside $\mathrm{Rf}$ in $P$. ginseng root were determined by coloring and $\mathrm{Rf}$ value by comparing them with the structures reported in the previous paper [19].

\section{Conclusion}

Fingerprint by eastern blotting method can open its application for the wide field of natural products, especially glycosides like solasodine glycosides and ginsenosides. An MAb having a wide cross-reactivity like anti-solamargine MAb can distinguish the total solasodine glycosides. On the other hand, a high specific MAb like anti-ginsenoside Rb1 MAb can be used for the detection of a single antigen compound.

When two kinds of MAbs can be used, the double-staining is possible as the staining system enhanced the separate staining of ginsenosides having protopanatriol or protopanaxadiol in a molecule. The staining color can monitor the pharmacological activity suggesting the stimulation activity and/or the depression affect for the CNS. Furthermore, the $\mathrm{Rf}$ value of solasodine glycosides and ginsenosides suggests the number of sugars attached to the aglycon. Both evidences make it possible to confirm which aglycon attaches and how many sugars combine to the aglycon resulting the confirmation of structure for glycosides. Therefore, the fingerprint stained by eastern blotting using MAb could provide a new strong methodology in the wide field of natural product investigation.

\section{References}

[1] R. Sakata, Y. Shoyama, and H. Murakami, "Production of monoclonal antibodies and enzyme immunoassay for typical adenylate cyclase activator, forskolin," Cytotechnology, vol. 16, no. 2, pp. 101-108, 1994.

[2] M. Ishiyama, Y. Shoyama, H. Murakami, and H. Shinohara, "Production of monoclonal antibodies and development of an ELISA for solamargine," Cytotechnology, vol. 18, no. 3, pp. 153-158, 1996.

[3] L. Xuan, H. Tanaka, Y. Xu, and Y. Shoyama, "Preparation of monoclonal antibody against crocin and its characterization," Cytotechnology, vol. 29, no. 1, pp. 65-70, 1999.

[4] H. Tanaka, Y. Goto, and Y. Shoyama, "Monoclonal antibody based enzyme immunoassay for marihuana (cannabinoid) compounds," Journal of Immunoassay, vol. 17, no. 4, pp. 321$342,1996$.

[5] Y. Shoyama, T. Fukada, and H. Murakami, "Production of monoclonal antibodies and ELISA for thebaine and codeine," Cytotechnology, vol. 19, no. 1, pp. 55-61, 1996.

[6] H. Tanaka, N. Fukuda, and Y. Shoyama, "Formation of monoclonal antibody against a major ginseng component, ginsenoside Rb1 and its characterization," Cytotechnology, vol. 29, no. 2, pp. 115-120, 1999.

[7] N. Fukuda, H. Tanaka, and Y. Shoyama, "Formation of monoclonal antibody against a major ginseng component, ginsenoside $\operatorname{Rg} 1$ and its characterization: monoclonal antibody for a ginseng saponin," Cytotechnology, vol. 34, no. 3, pp. 197-204, 2000.

[8] J. S. Kim, H. Tanaka, and Y. Shoyama, "Immunoquantitative analysis for berberine and its related compounds using monoclonal antibodies in herbal medicines," Analyst, vol. 129, no. 1, pp. 87-91, 2004.

[9] O. Morinaga, S. Nakajima, H. Tanaka, and Y. Shoyama, "Production of monoclonal antibodies against a major purgative component, sennoside B, their characterization and use in ELISA," Analyst, vol. 126, no. 8, pp. 1372-1376, 2001.

[10] Z. Lu, O. Morinaga, H. Tanaka, and Y. Shoyama, "A quantitative ELISA using monoclonal antibody to survey paeoniflorin and albiflorin in crude drugs and traditional Chinese herbal medicines," Biological and Pharmaceutical Bulletin, vol. 26, no. 6, pp. 862-866, 2003.

[11] H. Tanaka and Y. Shoyama, "Formation of a monoclonal antibody against glycyrrhizin and development of an ELISA," Biological and Pharmaceutical Bulletin, vol. 21, no. 12, pp. 1391-1393, 1998.

[12] S. J. Shan, H. Tanaka, and Y. Shoyama, "Enzyme-linked immunosorbent assay for glycyrrhizin using anti-glycyrrhizin monoclonal antibody and an eastern blotting technique for glucuronides of glycyrrhetic acid," Analytical Chemistry, vol. 73, no. 24, pp. 5784-5790, 2001.

[13] P. Loungratana, H. Tanaka, and Y. Shoyama, "Production of monoclonal antibody against ginkgolic acids in Ginkgo biloba Linn," The American Journal of Chinese Medicine, vol. 32, no. 1, pp. 33-48, 2004.

[14] K. Kido, K. Edakuni, O. Morinaga, H. Tanaka, and Y. Shoyama, "An enzyme-linked immunosorbent assay for aconitine-type alkaloids using an anti-aconitine monoclonal antibody," Analytica Chimica Acta, vol. 616, no. 1, pp. 109-114, 2008.

[15] K. Kido, O. Morinaga, Y. Shoyama, and H. Tanaka, "Quick analysis of baicalin in Scutellariae Radix by enzyme-linked immunosorbent assay using a monoclonal antibody," Talanta, vol. 77, no. 1, pp. 346-350, 2008.

[16] H. Tanaka, W. Putalun, C. Tsuzaki, and Y. Shoyama, "A simple determination of steroidal alkaloid glycosides by thin-layer chromatography immunostaining using monoclonal antibody against solamargine," FEBS Letters, vol. 404, no. 2-3, pp. 279282, 1997.

[17] H. Tanaka, N. Fukuda, and Y. Shoyama, "Eastern blotting and immunoaffinity concentration using monoclonal antibody for ginseng saponins in the field of traditional Chinese medicines," Journal of Agricultural and Food Chemistry, vol. 55, no. 10, pp. 3783-3787, 2007.

[18] O. Morinaga, H. Tanaka, and Y. Shoyama, "Detection and quantification of ginsenoside $\mathrm{Re}$ in ginseng samples by a chromatographic immunostaining method using monoclonal antibody against ginsenoside Re," Journal of Chromatography $B$, vol. 830, no. 1, pp. 100-104, 2006.

[19] N. Fukuda, S. Shan, H. Tanaka, and Y. Shoyama, "New staining methodology: eastern blotting for glycosides in the field of Kampo medicines," Journal of Natural Medicines, vol. 60, no. 1, pp. 21-27, 2006.

[20] O. Morinaga, S. Zhu, H. Tanaka, and Y. Shoyama, "Visual detection of saikosaponins by on-membrane immunoassay and estimation of traditional Chinese medicines containing Bupleuri radix," Biochemical and Biophysical Research Communications, vol. 346, no. 3, pp. 687-692, 2006.

[21] B. B. Shashi, P. S. Niranjan, N. G. Amar, R. Kasai, and O. Tanaka, "Steroidal alkaloids from Solanum khasianum: application of ${ }^{13} \mathrm{C}$ NMR spectroscopy to their structural elucidation," Phytochemistry, vol. 19, no. 9, pp. 2017-2020, 1980. 
[22] W. Putalun, F. Taura, W. Qing, H. Matsushita, H. Tanaka, and Y. Shoyama, "Anti-solasodine glycoside single-chain Fv antibody stimulates biosynthesis of solasodine glycoside in plants," Plant Cell Reports, vol. 22, no. 5, pp. 344-349, 2003.

[23] The Society for Korean Ginseng, In Understanding of Korean Ginseng, Seoul, Korea, 1995. 


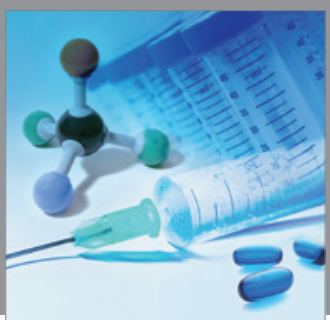

International Journal of

Medicinal Chemistry

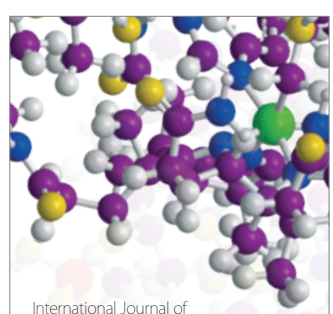

Carbohydrate Chemistry

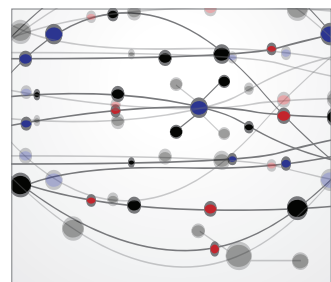

The Scientific World Journal
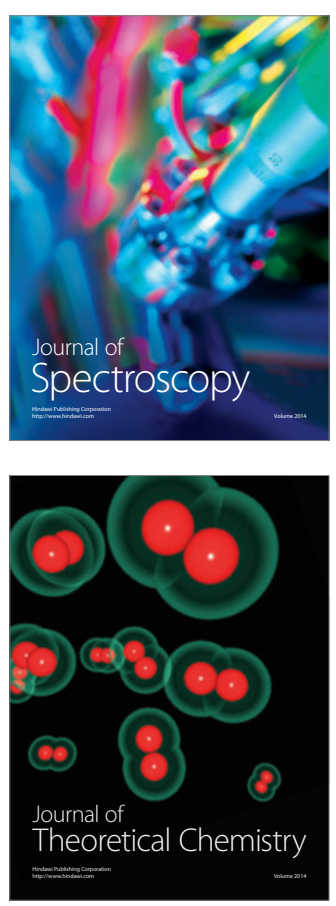
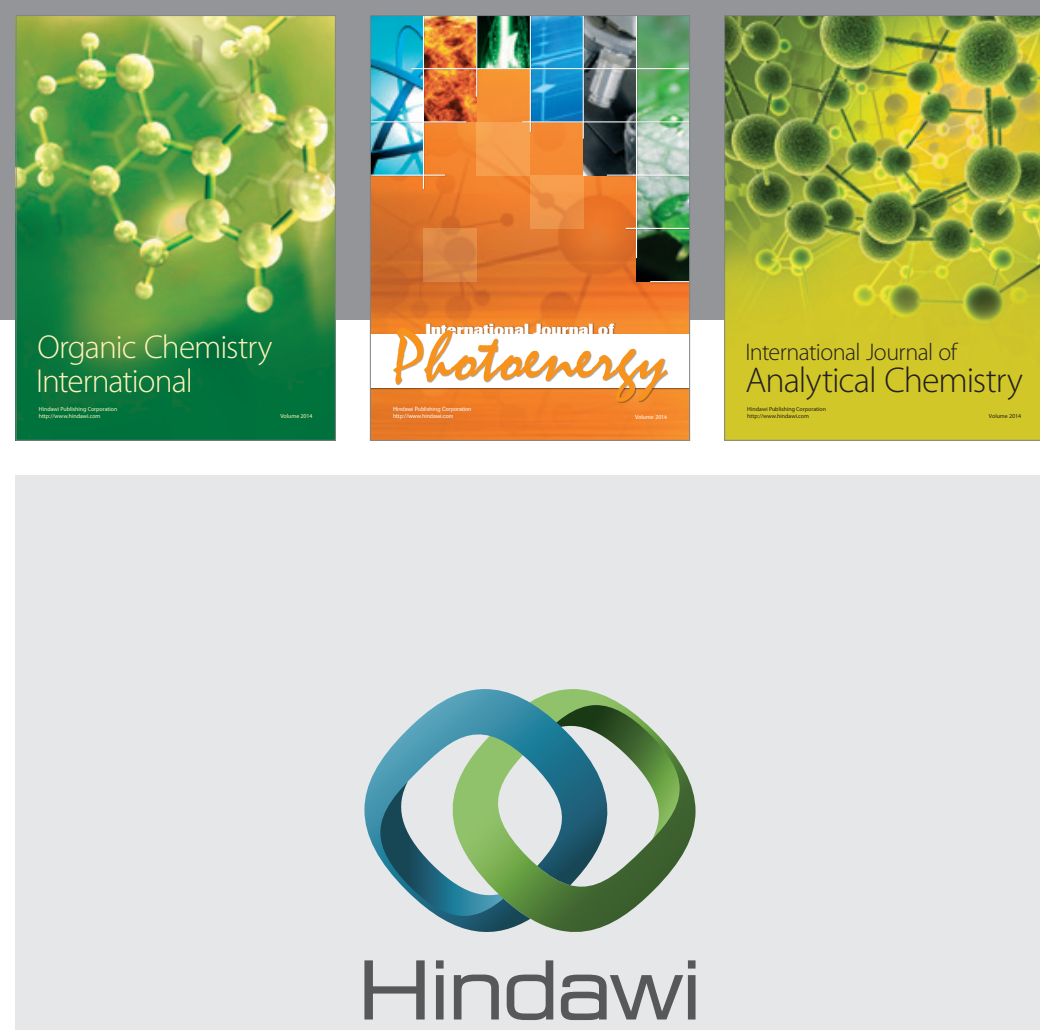

Submit your manuscripts at

http://www.hindawi.com
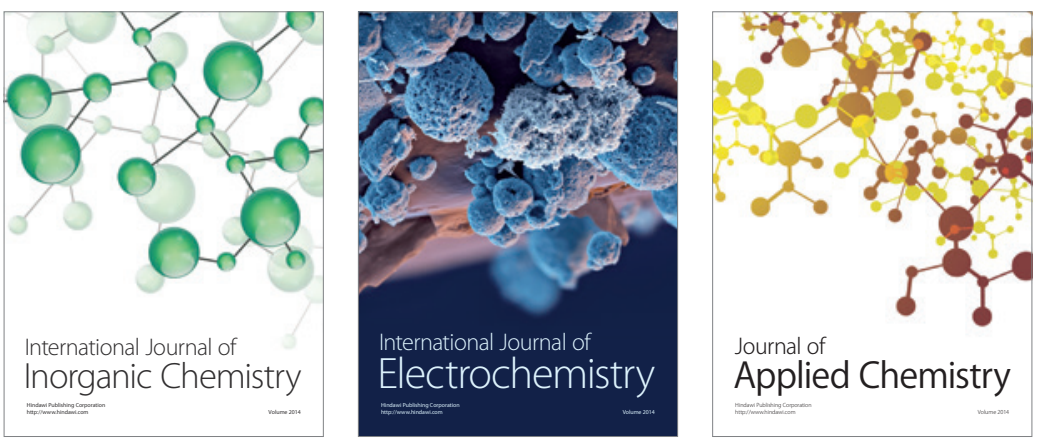

Journal of

Applied Chemistry
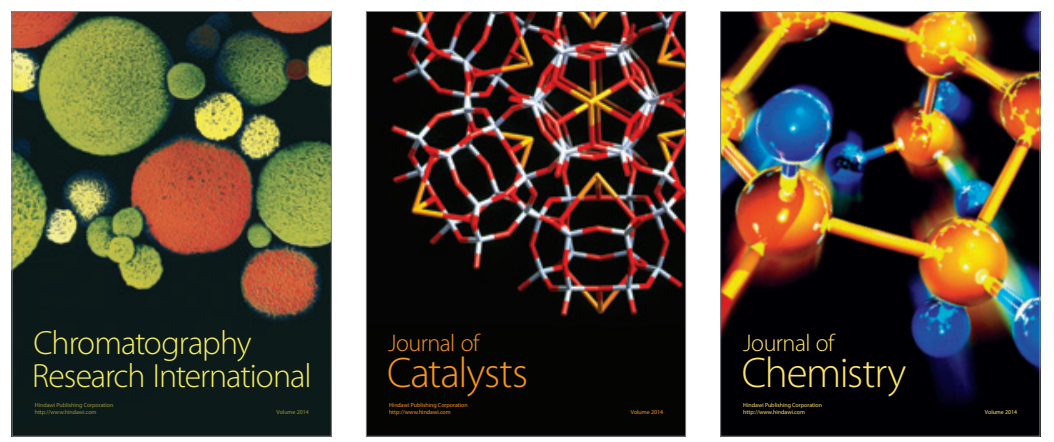
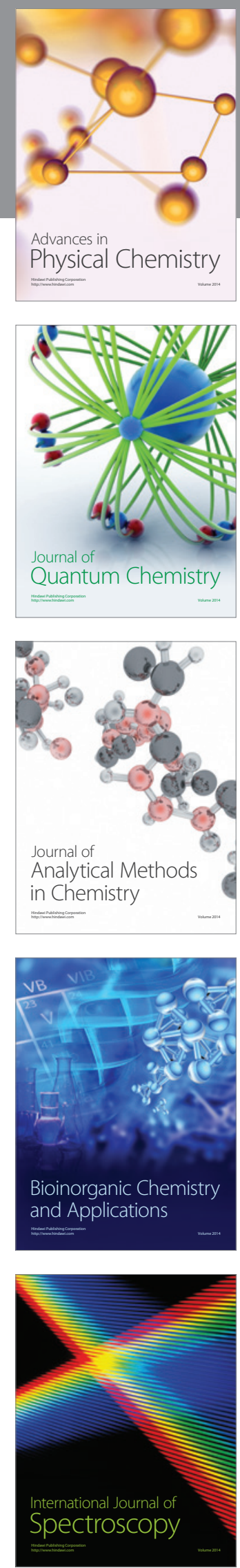